\title{
Dynamics of rogue waves on a multi-soliton background in a vector nonlinear Schrödinger equation
}

\author{
GUI MU ${ }^{\dagger}$, ZHENYUN QIN ${ }^{\ddagger *}$ AND ROGER GRIMSHAW § \\ $\dagger$ College of Mathematics and Information Science, Qujing Normal University, \\ Qujing Yunnan 655011, P.R. China \\ $\ddagger$ School of Mathematical Sciences and Key Laboratory of Mathematics \\ for Nonlinear Science, Fudan University, Shanghai 200433, P.R. China \\ $\S$ Department of Mathematical Sciences, Loughborough University, \\ Loughborough, Leics. LE11 3TU, UK.
}

\begin{abstract}
General higher order rogue waves of a vector nonlinear Schrödinger equation (Manakov system) are derived using a Darboux-dressing transformation with an asymptotic expansion method. The $N$ th order semi-rational solutions containing $3 N$ free parameters are expressed in separation of variables form. These solutions exhibit rogue waves on a multisoliton background. They demonstrate that the structure of rogue waves in this two-component system is richer than that in a one-component system. The study of our results would be of much importance in understanding and predicting rogue wave phenomena arising in nonlinear and complex systems, including optics, fluid dynamics, Bose-Einstein condensates and finance and so on.
\end{abstract}

Keywords. Vector nonlinear Schrödinger equations, rogue waves, Darboux-dressing transformation

AMS subject classification. 22E46, 53C35, 57S20

\section{Introduction}

It is well known that many nonlinear wave equations of physical interest support solitons, which are localised waves arising from a balance between dispersion and nonlinearity, and which can propagate steadily for a long time. Recently, over the last two decades, it has been recognised that another class of solutions, namely breathers, are also of fundamental importance. Breathers propagate steadily, and are localised in either time or space, while

${ }^{*}$ Corresponding author E-mail address: zyqin@fudan.edu.cn 
being periodic in either space or time. Further, due to their localisation properties, breathers have been invoked as models of rogue waves, also called freak waves, which are large amplitude waves which apparently appear without warning, and then disappear without trace. While they have been most often found in the context of water waves [1]-[3, they have also been found in other physical contexts such as optical fibres [4]-6].

The breather solutions of the focusing nonlinear Schrödinger equation (NLSE) have been widely invoked as models of rogue waves, see the references above and Akhmediev [7] for instance. The NLSE equation is integrable [8] and many kinds of exact solutions have been found. In particular, the Peregrine breather, [9] the Akhmediev breather (AB) [7] and the Kuznetsov-Ma breathers (KM) [10]-[11] have been associated with rogue waves, as the potential outcome of the modulational instability of a plane wave. AB is periodic in space and localized in time, while KM is periodic in time and localised in space. The Peregrine breather is especially considered as a rogue wave prototype because it is localised in both time and space, and so captures the fundamental features of rogue waves. Importantly it has a peak amplitude which is exactly three times the background. Also, it is the generic outcome of a wide class of modulated plane waves [12. When the spatial or temporal period is taken to be infinite, $\mathrm{AB}$ or KM become Peregrine breather in the limit. Peregrine breather of NLSE has been observed experimentally in water wave tanks [13] and also in nonlinear fibre optics [4]-[5].

The Peregrine breather is the lowest order item in a hierarchy of rational solutions for NLSE. Since these higher-order forms may also be invoked as models for rogue waves, with higher amplitudes, it is of interest to find explicit expressions for these higher-order rogue wave solutions. For example, Akhmediev [14] presented some low-order rational solutions using a Darboux transformation method. Ohta and Yang [15] obtained general high-order rogue waves in terms of determinants using the Hirota bilinear method. Guo [16]-[17] constructed the $N$-order rogue wave solutions using a generalized Darboux transformation. Dubard et al. [18] discussed the quasi-rational solutions via algebraicgeometry method. As well as for the NLSE, rational solutions have also been explored for some other nonlinear wave equations in [15], [19], [20]-223].

Here we shall extend a recent study of the integrable vector nonlinear Schrödinger equations (VNLSE), or Manakov system, by Baronio et al. [24] This can be expressed in dimensionless form as,

$$
\begin{aligned}
& i u_{1 t}+u_{1 x x}+2\left(\left|u_{1}\right|^{2}+\left|u_{2}\right|^{2}\right) u_{1}=0, \\
& i u_{2 t}+u_{2 x x}+2\left(\left|u_{1}\right|^{2}+\left|u_{2}\right|^{2}\right) u_{2}=0 .
\end{aligned}
$$

Here the subscripts $t, x$ stand for partial differentiation with resect to $t$, an evolution variable, and $x$, a spatial variable. The dependent variables $u_{1}, u_{2}$ represent wave envelopes, whose physical meaning depends on the particular context. In particular the system (11) has applications in nonlinear optics [25]-[27] and in Bose-Einstein condensates [28]-30]. Also note that Eqs. (1) correspond to the self-focusing (or anomalous dispersion) regime. The fundamental vector rogue wave solutions have been recently reported by several authors 24], 28], 31]. Then the first-, second- and third-order rogue wave 
solutions of VNLSE were explicitly presented [32]. Here our aim is to find the general $N$-th order rogue wave solutions. We will construct hierarchies of semi-rational solutions of the VNLSE (1), using an asymptotic expansion method. Our obtained solutions are an extension of the results of Baronio et al. [24] to higher-order rogue wave solutions, and the pure rational solutions of our results can be identified with the ones obtained by Zhai et al. [32. As the representations obtained are a combination of rational and exponential functions, some interesting structures can be observed, such as (1) the coexistence of higher-order rogue waves and bright-dark multi-soliton solutions, and (2) the coexistence of multi-rogue waves and bright-dark multi-soliton solutions. In general our obtained solutions indicate the complexity that can arise when rogue waves interact with solitons.

The paper is organised as follows. In section 2, The Lax pair and Darboux-dressing transformation of VNLSE (1) are briefly reviewed. In section 3 the dynamic behaviour of a family of fundamental rogue wave solutions is examined. Then in section 4 , we present some novel periodic breathers. In section 5, we use an asymptotic expansion method to obtain $N$-th order rogue wave solutions of VNLSE (1). Then, in section 6 , we give some examples to illustrate the a range of dynamic behaviour of our obtained rogue wave solutions. We conclude with a summary in section 7 .

\section{Asymptotic expansion of Darboux-dressing trans- formation}

The VNLSE (1) is integrable, and is a condition for the compatibility of the Lax pair,

$$
\begin{aligned}
& \Psi_{x}=\mathbf{U} \Psi=(i \lambda \sigma+Q) \Psi \\
& \Psi_{t}=\mathbf{V} \Psi=\left[2 i \lambda^{2} \sigma+2 \lambda Q+i \sigma\left(Q^{2}-Q_{x}\right)\right] \Psi .
\end{aligned}
$$

Here $\Psi(x, t)$ is a $3 \times 3$ matrix variable, $\lambda$ is the complex spectral parameter, $\sigma=$ $\operatorname{diag}(1,-1,-1)$ is a constant diagonal matrix and $Q=Q(x, t)$ is the $3 \times 3$ matrix variable

$$
Q=\left(\begin{array}{ccc}
0 & -u_{1}^{*} & -u_{2}^{*} \\
u_{1} & 0 & 0 \\
u_{2} & 0 & 0
\end{array}\right)
$$

It is straightforward to check the condition of compatibility between the two equation in (2)

$$
\Psi_{x t}=\Psi_{t x}
$$

leads directly to the VNLSE(1). 
A suitable Darboux-dressing transformation for the VNLSE (1) is given by [24] [33] for instance,

$$
\begin{aligned}
& \Psi[1]=D \Psi, \quad D=I+\frac{\left(\lambda_{1}^{*}-\lambda_{1}\right) P}{\lambda-\lambda_{1}^{*}}, \quad P=\frac{\psi_{0} \psi_{0}^{\dagger}}{\psi_{0}^{\dagger} \psi_{0}} \\
& \left(\begin{array}{c}
u_{1} \\
u_{2}
\end{array}\right)=\left(\begin{array}{c}
u_{10} \\
u_{20}
\end{array}\right)+\frac{2 i\left(\lambda_{1}^{*}-\lambda_{1}\right) r^{*}}{|r|^{2}+\left|s_{1}\right|^{2}+\left|s_{2}\right|^{2}}\left(\begin{array}{c}
s_{1} \\
s_{2}
\end{array}\right) .
\end{aligned}
$$

Here, $I=\operatorname{diag}(1,1,1), \quad \psi_{0}=\Psi\left(x, t, \lambda_{1}\right) Z_{0}=\left(r(x, t), s_{1}(x, t), s_{2}(x, t)\right)^{T}$ and $\Psi\left(x, t, \lambda_{1}\right)$ is the fundamental solution for the Lax equations (2) corresponding to $\lambda=\lambda_{1}$ and for the seed solutions $\mathbf{u}_{0}=\left(u_{10}, u_{20}\right)^{T}$ of the VNLSE (1). The constant parameter $\lambda_{1}$ is complex while $Z_{0}$ is an arbitrary nonzero complex 3-dimensional constant vector. Next, it is useful to note that the Darboux-dressing transformation (5) can be replaced with the alternative form

$$
\Psi[1]=T \Psi, \quad T=\lambda I+\Lambda, \quad \Lambda=-\lambda_{1}^{*} I+\left(\lambda_{1}^{*}-\lambda_{1}\right) P, \quad P=\frac{\psi_{0} \psi_{0}^{\dagger}}{\psi_{0}^{\dagger} \psi_{0}} .
$$

Since $T$ is also a Darboux transformation of NLSE, it follows that

$$
T_{x}+T \mathbf{U}-\mathbf{U}_{1} T=0, \quad T_{t}+T \mathbf{V}-\mathbf{V}_{1} T=0,
$$

The matrices $\mathbf{U}_{1}$ and $\mathbf{V}_{1}$ are obtained by replacing $Q$ with $Q_{1}$ in $\mathbf{U}$ and $\mathbf{V}$, respectively. Then inserting (7) into (8) we find that

$$
\begin{aligned}
& \Lambda_{x}+\Lambda Q_{0}-Q_{1} \Lambda=0, \\
& \Lambda_{t}+i \Lambda \sigma\left(Q_{0}^{2}-Q_{0 x}\right)-i \sigma\left(Q_{1}^{2}-Q_{1 x}\right) \Lambda=0, \\
& \text { and } Q_{1}=Q_{0}+i \Lambda \sigma-i \sigma \Lambda,
\end{aligned}
$$

In general, for a Darboux transformation, the zero seed solution allows for the construction of a hierarchy of multisoliton solutions, while a plane wave seed solution results in a hierarchy of breather-type solutions related to modulation instability. However, here we note that

$$
\left.T\right|_{\lambda=\lambda_{1}} \psi_{0}=\left(\lambda_{1} I+\Lambda_{1}\right) \psi_{0}=0
$$

This means that the Darboux-dressing transformation (7) cannot be iterated continuously for the same spectral parameter. In order to eliminate this limitation, we introduce the following expansion theorem which can then be used to produce new solutions for the same spectral parameter. 
Theorem Let $\Psi\left[\lambda_{1}(1+\delta)\right]$ be a solution of the Lax pair system (2) corresponding to the spectral parameter $\lambda_{1}(1+\delta)$ and a seed solution $\mathbf{u}_{0}=\left(u_{1}[0], u_{2}[0]\right)^{T}$. If $\Psi\left[\lambda_{1}(1+\delta)\right]$ has an expansion at $\lambda_{1}$

$$
\begin{aligned}
& \Psi\left[\lambda_{1}(1+\delta)\right]=\Psi_{0}+\Psi_{1} \delta+\Psi_{2} \delta^{2}+\Psi_{3} \delta^{3}+\cdots, \\
& \text { then } \quad \psi[n]=\left(r[n], s_{1}[n], s_{2}[n]\right)^{T}=\lambda_{1} \psi[n-1]+T[n] \Upsilon[n-1], \quad n \geq 1, \\
& \psi[0]=\left(r[0], s_{1}[0], s_{2}[0]\right)^{T}=\Psi_{0}, \\
& \Upsilon[n-1]=\psi[n-1]\left(\Psi_{j} \rightarrow \Psi_{j+1}\right), \quad j=0,1,2, \cdots \\
& \text { where } \quad T[n]=\lambda_{1} I+\Lambda[n], \quad \Lambda[n]=-\lambda_{1}^{*}+\left(\lambda_{1}^{*}-\lambda_{1}\right) P[n], \\
& \text { and } \quad P[n]=\frac{\psi[n-1] \psi[n-1]^{\dagger}}{\psi[n-1]^{\dagger} \psi[n-1]},
\end{aligned}
$$

are solutions of the Lax pair system (2) corresponding to the same spectral parameter $\lambda_{1}$ and solution $\mathbf{u}_{n} \equiv\left(\begin{array}{l}u_{1}[n] \\ u_{2}[n]\end{array}\right)$

$$
\mathbf{u}_{n}=\left(\begin{array}{c}
u_{1}[n-1] \\
u_{2}[n-1]
\end{array}\right)+\frac{21\left(\lambda_{1}^{*}-\lambda_{1}\right) r[n-1]^{*}}{|r[n-1]|^{2}+\left|s_{1}[n-1]\right|^{2}+\left|s_{2}[n-1]\right|^{2}}\left(\begin{array}{c}
s_{1}[n-1] \\
s_{2}[n-1]
\end{array}\right) .
$$

Remark: In the above Theorem, the denotation $\psi[n]\left(\Psi_{j} \rightarrow \Psi_{j+1}\right)$ means that $\Psi_{j}(j=$ $1,2, \cdots n)$ are replaced correspondingly by $\Psi_{j+1}$ but $T[j](j=1,2, \cdots n)$ are left unchanged in $\psi[n]$. In addition, we introduce the denotation

$$
\Phi[N]=\Upsilon[N]\left(\Psi_{j} \rightarrow \Psi_{j+1}\right),
$$

The meaning of $\Upsilon[N]\left(\Psi_{j} \rightarrow \Psi_{j+1}\right)$ is same as $\psi[n]\left(\Psi_{j} \rightarrow \Psi_{j+1}\right)$. Concretely, let us illustrate these denotations by two simplest examples: for $n=0, \psi[0]=\Psi_{0}, \Upsilon[0]=$ $\Psi_{1}, \Phi[0]=\Psi_{2}$. For $n=1, \psi[1]=\lambda_{1} \psi[0]+T[1] \Upsilon[0]=\lambda_{1} \Psi_{0}+T[1] \Psi_{1}, \Upsilon[1]=\lambda_{1} \Upsilon[0]+$ $T[1] \Phi[0]=\lambda_{1} \Psi_{1}+T[1] \Psi_{2}, \Phi[1]=\lambda_{1} \Psi_{2}+T[1] \Psi_{3}$.

Generally, employing the iteration relation (13) and with the help of the following expansion

$$
\begin{aligned}
P(\lambda) & =\left(\lambda_{1}+T[n]\right)\left(\lambda_{1}+T[n-1]\right) \cdots\left(\lambda_{1}+T[1]\right) \\
& =f_{0} \lambda_{1}^{n}+f_{1} \lambda_{1}^{n-1}+f_{2} \lambda_{1}^{n-2}+\cdots+f_{n}=\sum_{j=0}^{n} g_{j},
\end{aligned}
$$

with

$$
\begin{aligned}
& g_{j}=\lambda_{1}^{n-j} f_{j}, \quad j=0,1, \cdots, n . \\
& f_{0}=1, \quad f_{1}=T[1]+T[2]+\cdots+T[n], \quad \cdots, \\
& f_{n}=T[n] T[n-1] \cdots T[1] .
\end{aligned}
$$


Then $\psi[n]$ could be rewritten in a more explicit form

$$
\psi[n]=g_{0} \Psi_{0}+g_{1} \Psi_{1}+g_{2} \Psi_{2}+\cdots+g_{n} \Psi_{n},
$$

It is natural to obtain

$$
\Upsilon[n]=g_{0} \Psi_{1}+g_{1} \Psi_{2}+g_{2} \Psi_{3}+\cdots+g_{n} \Psi_{n+1},
$$

and

$$
\Phi[n]=g_{0} \Psi_{2}+g_{1} \Psi_{3}+g_{2} \Psi_{4}+\cdots+g_{n} \Psi_{n+2},
$$

Based on these preparations, we turn to the proof of Theorem.

Proof: Substituting (12) into (2) and equation to zero, the coefficients of $\delta^{j} \quad(j=$ $0,1, \cdots)$, we arrive at

$$
\begin{aligned}
& \Psi_{0 x}=U_{0} \Psi_{0}, \\
& \Psi_{j x}=i \lambda_{1} \sigma \Psi_{j-1}+U_{0} \Psi_{j},(j \geq 1)
\end{aligned}
$$

and

$$
\begin{aligned}
\Psi_{0 t} & =V_{0} \Psi_{0}, \\
\Psi_{1 t} & =V_{0} \Psi_{1}+\left(4 i \lambda_{1}^{2} \sigma+2 \lambda_{1} Q_{0}\right) \Psi_{0}, \\
\Psi_{j t} & =V_{0} \Psi_{j}+\left(4 i \lambda_{1}^{2} \sigma+2 \lambda_{1} Q_{0}\right) \Psi_{j-1} \\
& +21 \lambda_{1}^{2} \sigma \Psi_{j-2},(j \geq 2)
\end{aligned}
$$

with

$$
U_{0}=i \lambda_{1} \sigma+Q_{0}, \quad V_{0}=2 i \lambda_{1}^{2} \sigma+2 \lambda_{1} Q_{0}+i \sigma\left(Q_{0}^{2}-Q_{0 x}\right),
$$

From the first equation of (18) and $(19)$, we conclude that $\psi[0]=\left(r[0], s_{1}[0], s_{2}[0]\right)^{T}=\Psi_{0}$ is a solution of the the linear systems (2) with $\mathbf{u}_{0}$ and $\lambda=\lambda_{1}$. Thus, we can construct a Darboux transformation as in (7) above

$$
\begin{aligned}
& \Psi[1]=T[1] \Psi, \quad T[1]=\lambda I+\Lambda[1], \\
& \Lambda[1]=-\lambda_{1}^{*} I+\left(\lambda_{1}^{*}-\lambda_{1}\right) P[1], \quad P[1]=\frac{\psi[0] \psi[0]^{\dagger}}{\psi[0]^{\dagger} \psi[0]}, \\
& \quad \text { and then }\left.\quad T\right|_{\lambda=\lambda_{1}} \psi[0]=T[1] \Psi_{0}=0 .
\end{aligned}
$$

Meanwhile, the corresponding solution of the VNLSE is given by

$$
\mathbf{u}_{1} \equiv\left(\begin{array}{c}
u_{1}[1] \\
u_{2}[1]
\end{array}\right)=\left(\begin{array}{c}
u_{1}[0] \\
u_{2}[0]
\end{array}\right)+\frac{2 i\left(\lambda_{1}^{*}-\lambda_{1}\right) r[0]^{*}}{|r[0]|^{2}+\left|s_{1}[0]\right|^{2}+\left|s_{2}[0]\right|^{2}}\left(\begin{array}{c}
s_{1}[0] \\
s_{2}[0]
\end{array}\right)
$$


Thus we have shown that the Theorem holds for $n=0$.

Next we use mathematical induction for integers $n \geq 1$. Assume the Theorem holds for $n \leq N$, that is,

$$
\begin{aligned}
& \psi[n]_{x}=\left(i \lambda_{1} \sigma+Q_{n}\right) \psi[n], \\
& \psi[n]_{t}=\left[2 i \lambda^{2} \sigma+2 \lambda Q_{n}+i \sigma\left(Q_{n}^{2}-Q_{n x}\right)\right] \psi[n],
\end{aligned}
$$

with $\psi[n]=\lambda_{1} \psi[n-1]+T[n] \Upsilon[n-1], \Upsilon[n-1]=\psi[n-1]\left(\Psi_{j} \rightarrow \Psi_{j+1}\right)$.

Employing the following properties

$$
\begin{aligned}
& \psi[N-1]_{x}=\left(i \lambda_{1} \sigma+Q_{N-1}\right) \psi[N-1], \\
& T[N]_{x}=\Lambda[N]_{x}=Q_{N} \Lambda[N]-\Lambda[N] Q_{N-1}, \quad T[N] \psi[N-1]=0, \\
& Q_{N}=Q_{N-1}+i \Lambda[N] \sigma-i \sigma \Lambda[N],
\end{aligned}
$$

the left side of 23 is given by

$$
\begin{aligned}
& \left(\lambda_{1} \psi[N-1]+T[N] \Upsilon[N-1]\right)_{x} \\
& =\lambda_{1} \psi[N-1]_{x}+T[N]_{x} \Upsilon[N-1]+T[N] \Upsilon[N-1]_{x}, \\
& =\lambda_{1}\left(i \lambda_{1} \sigma+Q_{N-1}\right) \psi[N-1]+\left(Q_{N} \Lambda_{N}-\Lambda[N] Q_{N-1}\right) \Upsilon[N-1]+T[N] \Upsilon[N-1]_{x},
\end{aligned}
$$

Comparing this with the right side of (23), we get that

$$
\begin{aligned}
& T[N] \Upsilon[N-1]_{x}=\left(\lambda_{1}\left(i \lambda_{1} \sigma+Q_{N}\right)+i \lambda_{1} \sigma \Lambda[N]+\Lambda[N] Q_{N-1}\right) \Upsilon[N-1] \\
& \quad+\lambda_{1}\left(Q_{N}-Q_{N-1}\right) \psi[N-1] \\
& =\left(i \lambda_{1}^{2} \sigma+\lambda_{1}\left(Q_{N-1}+i \Lambda[N] \sigma-i \sigma \Lambda[N]\right)+i \lambda_{1} \sigma \Lambda[N]+\Lambda[N] Q_{N-1}\right) \Upsilon[N-1] \\
& \quad+\lambda_{1}(i \Lambda[N] \sigma-i \sigma \Lambda[N]) \psi[N-1] \\
& =T[N]\left(i \lambda_{1} \sigma+Q_{N-1}\right) \Upsilon[N-1]+i \lambda_{1} T[N] \sigma \psi[N-1],
\end{aligned}
$$

Factoring out the factor $T[N]$ in (28), we arrive at

$$
\Upsilon[N-1]_{x}=\left(i \lambda_{1} \sigma+Q_{N-1}\right) \Upsilon[N-1]+i \lambda_{1} \sigma \psi[N-1],
$$

Furthermore, from (16), (17) and (18), we have

$$
\begin{aligned}
\Upsilon[n]_{x} & =\sum_{j=0}^{n}\left(g_{j x} \Psi_{j+1}+g_{j} \Psi_{j+1, x}\right) \\
& =\sum_{j=0}^{n}\left(i \lambda_{1} \sigma \Psi_{j}+\left(g_{j x}+U_{0}\right) \Psi_{j+1}\right),
\end{aligned}
$$


and

$$
\begin{aligned}
\Phi[n]_{x} & =\sum_{j=0}^{n}\left(g_{j x} \Psi_{j+2}+g_{j} \Psi_{j+2, x}\right) \\
& =\sum_{j=0}^{n}\left(i \lambda_{1} \sigma \Psi_{j+1}+\left(g_{j x}+U_{0}\right) \Psi_{j+2}\right),
\end{aligned}
$$

For an arbitrary nonnegative integer $n$, by a comparison (30) with (31), it indicate that $\Phi[n]_{x}$ can be obtained directly after replacing $\Psi_{j}$ by $\Psi_{j+1}$ in $\Upsilon[n]_{x}$. As a result, from (29), we immediately get

$$
\Phi[N-1]_{x}=\left(i \lambda_{1} \sigma+Q_{N-1}\right) \Phi[N-1]+i \lambda_{1} \sigma \Upsilon[N-1],
$$

Now, with the help of (29) and (32), it is derived that

$$
\begin{aligned}
\Upsilon & {[N]_{x}=\left(\lambda_{1} \Upsilon[N-1]+T[N] \Phi[N-1]\right)_{x} } \\
= & \lambda_{1} \Upsilon[N-1]_{x}+T[N]_{x} \Phi[N-1]+T[N] \Phi[N-1]_{x} \\
= & \lambda_{1}\left(\left(i \lambda_{1} \sigma+Q_{N-1}\right) \Upsilon[N-1]+i \lambda_{1} \sigma \psi[N-1]\right)+\left(Q_{N} \Lambda[N]-\Lambda[N] Q_{N-1}\right) \Phi[N-1] \\
& +T[N]\left(\left(i \lambda_{1} \sigma+Q_{N-1}\right) \Phi[N-1]+i \lambda_{1} \sigma \Upsilon[N-1]\right) \\
= & \left(i \lambda_{1}^{2} \sigma+\lambda_{1} Q_{N-1}+i \lambda_{1} T[N] \sigma\right) \Upsilon[N-1]+i \lambda_{1}^{2} \sigma \psi[N-1] \\
& +\left(Q_{N} \Lambda[N]-\Lambda[N] Q_{N-1}+i \lambda_{1} T[N] \sigma+T[N] Q_{N-1}\right) \Phi[N-1] \\
= & \left(i \lambda_{1}^{2} \sigma+\lambda_{1} Q_{N}+i \lambda_{1} \sigma \Lambda[N]+i \lambda_{1}^{2} \sigma\right) \Upsilon[N-1]+i \lambda_{1}^{2} \sigma \psi[N-1] \\
& +\left(Q_{N} \Lambda[N]+i \lambda_{1} T[N] \sigma+\lambda_{1} Q_{N-1}\right) \Phi[N-1] \\
= & \left(i \lambda_{1}^{2} \sigma+\lambda_{1} Q_{N}\right) \Upsilon[N-1]+i \lambda_{1} \sigma \psi[N] \\
& +\left(Q_{N} \Lambda[N]+i \lambda_{1}^{2} \sigma+\lambda_{1} Q_{N}+i \lambda_{1} \sigma \Lambda[N]\right) \Phi[N-1] \\
= & \left(i \lambda_{1} \sigma+Q_{N}\right)\left(\lambda_{1} \Upsilon[N-1]+T[N] \Phi[N-1]\right)+i \lambda_{1} \sigma \psi[N] \\
= & \left(i \lambda_{1} \sigma+Q_{N}\right) \Upsilon[N]+i \lambda_{1} \sigma \psi[N]
\end{aligned}
$$

The assumption (23) allows us to construct the next step of the Darboux dressing transformation $T[N+1]$ which satisfies

$$
\begin{aligned}
& T[N+1]_{x}=\Lambda[N+1]_{x}=Q_{N+1} \Lambda[N+1]-\Lambda[N+1] Q_{N}, \\
& T[N+1] \psi[N]=\left(\lambda_{1} I+\Lambda[N+1]\right) \psi[N]=0, \\
& Q_{N+1}=Q_{N}+i \Lambda[N+1] \sigma-i \sigma \Lambda[N+1],
\end{aligned}
$$

Therefore, using (33) and (34), it follows that

$$
\begin{aligned}
T & {[N+1] \Upsilon[N]_{x}=\left(\lambda_{1} I+\Lambda[N+1]\right)\left(\left(i \lambda_{1} \sigma+Q_{N}\right) \Upsilon[N]+i \lambda_{1} \sigma \psi[N]\right) } \\
= & \left(\lambda_{1} I+\Lambda[N+1]\right)\left(i \lambda_{1} \sigma+Q_{N}\right) \Upsilon[N]+i \lambda_{1}\left(\lambda_{1} I+\Lambda[N+1]\right) \sigma \psi[N] \\
= & \left(i \lambda_{1}^{2} \sigma+\lambda_{1} Q_{N}+i \lambda_{1} \Lambda[N+1] \sigma+\Lambda[N+1] Q_{N}\right) \Upsilon[N] \\
& +i \lambda_{1}(-\sigma \Lambda[N+1]+\Lambda[N+1] \sigma) \psi[N] \\
= & \left(\lambda_{1}\left(i \lambda_{1} \sigma+Q_{N+1}\right)+i \lambda_{1} \sigma \Lambda[N+1]+\Lambda[N+1] Q_{N}\right) \Upsilon[N] \\
& +\lambda_{1}\left(Q_{N+1}-Q_{N}\right) \psi[N],
\end{aligned}
$$


Next, we need to show that

$$
\psi[N+1]_{x}=\left(i \lambda_{1} \sigma+Q_{N+1}\right) \psi[N+1]
$$

Indeed, using (23)- 35$)$, we get that

$$
\begin{aligned}
\psi & {[N+1]_{x}=\left(\lambda_{1} \psi[N]+T[N+1] \Upsilon[N]\right)_{x} } \\
= & \lambda_{1} \psi[N]_{x}+\Lambda[N+1]_{x} \Upsilon[N]+T[N+1] \Upsilon[N]_{x} \\
= & \lambda_{1}\left(i \lambda_{1} \sigma+Q_{N}\right) \psi[N]+\left(Q_{N+1} \Lambda[N+1]-\Lambda[N+1] Q_{N}\right) \Upsilon[N] \\
& +\left(\lambda_{1}\left(i \lambda_{1} \sigma+Q_{N+1}\right)+i \lambda_{1} \sigma \Lambda[N+1]+\Lambda[N+1] Q_{N}\right) \Upsilon[N] \\
& +\lambda_{1}\left(Q_{N+1}-Q_{N}\right) \psi[N] \\
= & \lambda_{1}\left(i \lambda_{1} \sigma+Q_{N+1}\right) \psi[N]+\left(i \lambda_{1} \sigma+Q_{N+1}\right)\left(\lambda_{1}+\Lambda[N+1]\right) \Upsilon[N] \\
= & \left(i \lambda_{1} \sigma+Q_{N+1}\right)\left(\lambda_{1} \psi[N]+T[N+1] \Upsilon[N]\right) \\
= & \left(i \lambda_{1} \sigma+Q_{N+1}\right) \psi[N+1],
\end{aligned}
$$

Similarly, for the temporal flow, we can show that

$$
\psi[N+1]_{t}=\left[2 i \lambda^{2} \sigma+2 \lambda Q_{N+1}+i \sigma\left(Q_{N+1}^{2}-Q_{N+1, x}\right)\right] \psi[N+1] .
$$

This completes the proof by induction.

In addition, by a slight adjustment of the above proof of our Theorem, it will provide a justifiable way to prove the Theorem 2 in Guo et al. [16].

\section{Exact breather solutions of the Lax pair system}

Before using the expansion Theorem to construct higher-order rogue wave solutions, we use the Darboux-dressing transformation (2) to construct a new class of breather solutions. It is readily shown that the VNLSE (1) admit the plane wave background solution,

$$
u_{10}=a_{1} e^{i \varphi}, u_{20}=a_{2} e^{i \varphi}
$$

$$
\text { where } \varphi=k x+\left(2 \omega^{2}-k^{2}\right) t, \quad \omega=\sqrt{a_{1}^{2}+a_{2}^{2}},
$$

where $a_{1}$ and $a_{2}$ are arbitrary parameters which, without loss of generality, are taken as real, and $k$ is a wavenumber.

The corresponding solution of the Lax system (2) is sought in the form

$$
\begin{gathered}
\Psi=\left(r(x, t), s_{1}(x, t), s_{2}(x, t)\right)^{T}=A F G z, \\
F=\exp (i \Theta x), \quad G=\exp (i \Omega t), \quad A=\operatorname{diag}\left(1, e^{i \varphi}, e^{1 \varphi}\right),
\end{gathered}
$$


and $z=\left(\mu_{1}, \mu_{2}, \mu_{3}\right)^{T}$ is an arbitrary complex vector, and $v=\Psi z$. Here, it is required that the constant matrices $\Theta$ and $\Omega$ satisfy

$$
[\Theta, \Omega]=\Theta \Omega-\Omega \Theta=0 .
$$

Inserting (39) into (2) yields

$$
A_{x}+i A \Theta-\mathbf{U} A=0, \quad A_{t}+i A \Omega-\mathbf{V} A=0,
$$

Solving the conditions (41) and (42), we obtain

$$
\begin{aligned}
& \Theta=\left(\begin{array}{ccc}
\lambda & i a_{1} & i a_{2} \\
-i a_{1} & -\lambda-k & 0 \\
-i a_{2} & 0 & -\lambda-k
\end{array}\right), \\
& \Omega=\Theta^{2}+2 \lambda \Theta-\lambda^{2}-2 \omega^{2},
\end{aligned}
$$

Then the exponential matrices $F$ in $(39)$ can be written as

$$
F=\frac{1}{2 \tau} \exp (-i k x / 2)\left(\begin{array}{ccc}
\eta_{1} & \eta_{2} & \eta_{3} \\
-\eta_{2} & \eta_{4} & \eta_{5} \\
-\eta_{3} & \eta_{5} & \eta_{6}
\end{array}\right)
$$

where

$$
\begin{aligned}
\eta_{1} & =2 \tau \cosh (\tau x)+i \mu \sinh (\tau x), \quad \eta_{2}=-2 a_{1} \sinh (\tau x), \quad \eta_{3}=-2 a_{2} \sinh (\tau x), \\
\eta_{4} & =a_{1}^{2} \omega^{-2}[2 \tau \cosh (\tau x)-i \mu \sinh (\tau x)]+2 a_{2}^{2} \tau \omega^{-2} e^{-i \mu x / 2}, \\
\eta_{5} & =a_{1} a_{2} \omega^{-2}\left[2 \tau \cosh (\tau x)-i \mu \sinh (\tau x)-2 \tau e^{-i \mu x / 2}\right], \\
\eta_{6} & =a_{2}^{2} \omega^{-2}[2 \tau \cosh (\tau x)-i \mu \sinh (\tau x)]+2 a_{1}^{2} \tau \omega^{-2} e^{-i \mu x / 2}, \\
\tau & =\frac{i}{2} \sqrt{\mu^{2}+4 \omega^{2}}, \quad \mu=2 \lambda+k .
\end{aligned}
$$

Similarly the exponential matrices $G$ in 39 can be written as

$$
G=\frac{1}{2 \xi} \exp \left(i k^{2} t / 2-i \omega^{2} t\right)\left(\begin{array}{ccc}
\kappa_{1} & \kappa_{2} & \kappa_{3} \\
-\kappa_{2} & \kappa_{4} & \kappa_{5} \\
-\kappa_{3} & \kappa_{5} & \kappa_{6}
\end{array}\right)
$$

where

$$
\begin{aligned}
& \kappa_{1}=2 \xi \cosh (\xi t)+i \mu \nu \sinh (\xi t), \quad \kappa_{2}=-2 a_{1} \nu \sinh (\xi t), \quad \kappa_{3}=-2 a_{2} \nu \sinh (\xi t), \\
& \kappa_{4}=a_{1}^{2} \omega^{-2}[2 \xi \cosh (\xi t)-i \mu \nu \sinh (\xi t)]+2 a_{2}^{2} \xi \omega^{-2} e^{-i\left(\omega^{2} t+\mu \nu t / 2\right)}, \\
& \kappa_{5}=a_{1} a_{2} \omega^{-2}\left[2 \xi \cosh (\xi t)-i \mu \nu \sinh (\xi t)-2 \xi e^{-i\left(\omega^{2} t+\mu \nu t / 2\right)}\right], \\
& \kappa_{6}=a_{2}^{2} \omega^{-2}[2 \xi \cosh (\xi t)-i \mu \nu \sinh (\xi t)]+2 a_{1}^{2} \xi \omega^{-2} e^{-i\left(\omega^{2} t+\mu \nu t / 2\right)}, \\
& \xi=\nu \tau, \quad \nu=2 \lambda-k .
\end{aligned}
$$



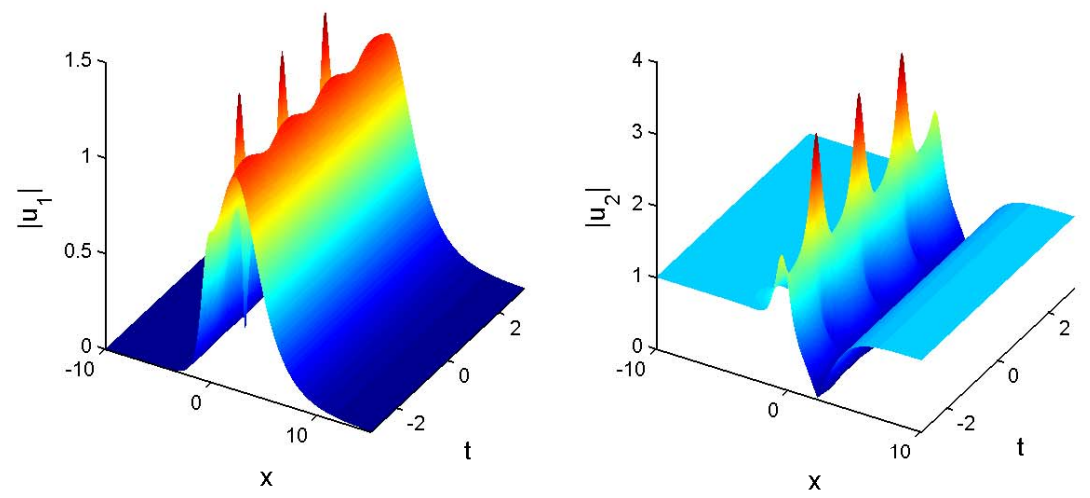

Figure 1: (Color online) Bright-dark temporally periodic breather of the VNLSE (1) for parameters $a_{1}=0, a_{2}=1, k=0, \lambda=\frac{5}{4} i, \mu_{1}=0, \mu_{2}=1, \mu_{3}=2$.
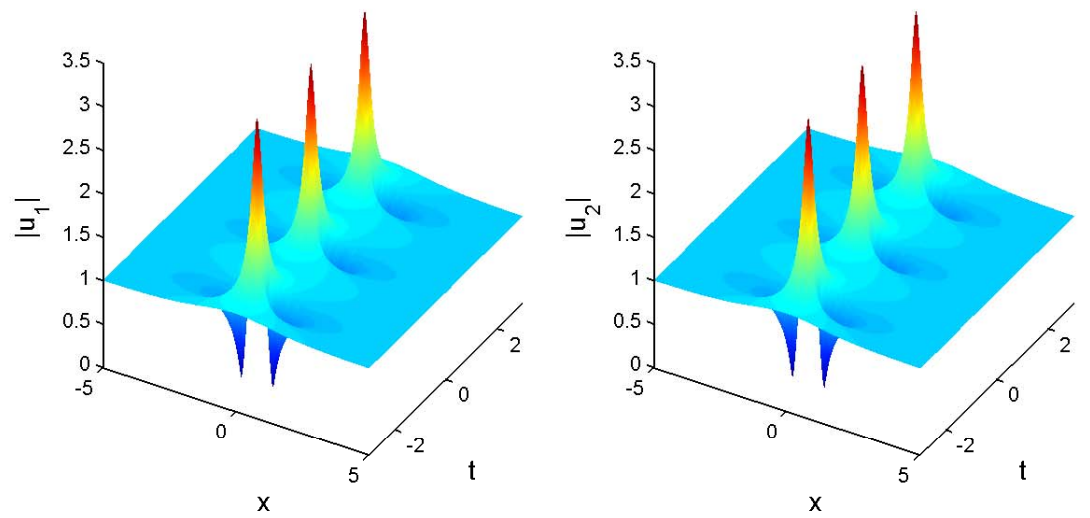

Figure 2: (Color online) A typical temporal periodic breather of the VNLSE (1) for parameters $a_{1}=1, a_{2}=1, k=0, \lambda=\frac{3}{2} i, \mu_{1}=1, \mu_{2}=1, \mu_{3}=1$. 

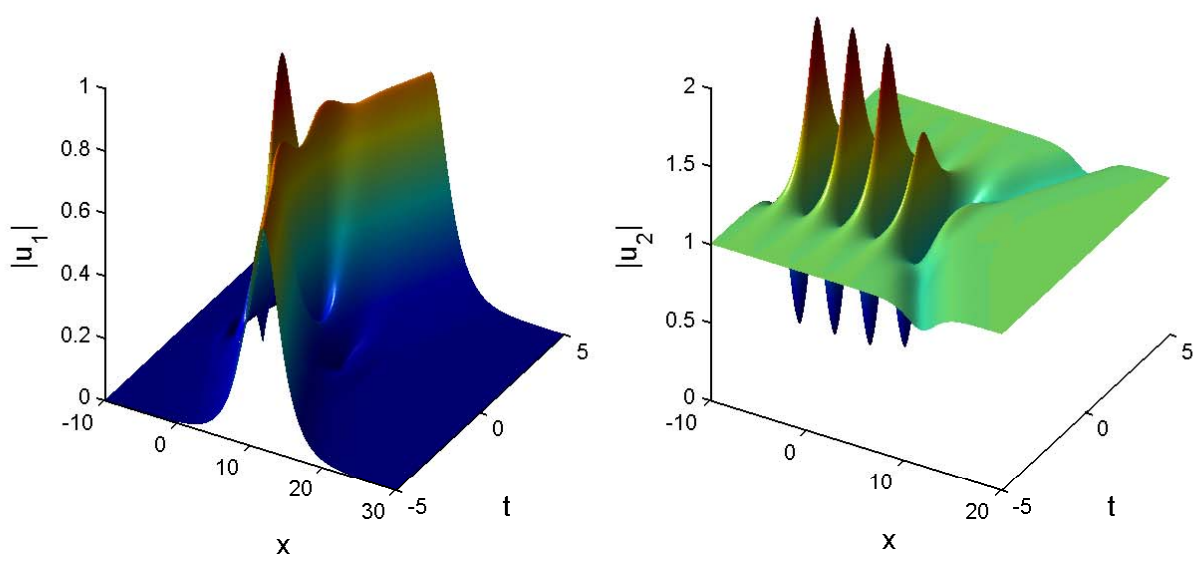

Figure 3: (Color online) Bright-dark spatially periodic breather of the VNLSE (1) for parameters $a_{1}=0, a_{2}=1, \lambda=\frac{i}{2}, \mu_{1}=1, \mu_{2}=1, \mu_{3}=5$.

Next, using the plane wave (38) as the seed solution in the Darboux transformation (2.5) we can construct a new exact solution of the VNLSE (1) composed of hyperbolic functions and exponential functions. Since their full expressions are cumbersome, we omit them here. In particular, we note that when $\lambda=i h,-2 \omega<h<2 \omega$, the solutions become spatially periodic. On the other hand, when $\lambda=i h, h>2 \omega$ or $h<-2 \omega$, the solutions become temporally periodic. Figures 1 and 2 show two different temporally periodic breathers while Figures 3 and 4 show two spatially periodic breathers. Due to the exponential functions in these solutions, the dynamic features of these breathers are different from the cases studied by Forest [27].

Further,the exponential functions in these solutions can be eliminated if the components of the vector $Z_{0}$ satisfy the condition

$$
a_{1} \mu_{3}-a_{2} \mu_{2}=0
$$

Under this constraint, the dynamic behavior will then be similar to those of AB and KM in the NLSE (see Figures 2 and 4). It is well known that the Peregrine breather is a limiting case of $\mathrm{AB}$ and $\mathrm{KM}$ when the period of either solution becomes infinite, and the same process could be employed here.

When $\lambda=-\frac{k}{2} \pm i \omega$, the exponential $\exp (i \Lambda x+i \Omega t)$ becomes a combination of exponential and polynomial functions of $x$ and $t$. In particular, taking $\lambda=-\frac{k}{2}+i \omega$, we arrive at a family of the solution with a wavenumber $k$

$$
\left(\begin{array}{l}
u_{1} \\
u_{2}
\end{array}\right)=e^{i \theta}\left(\begin{array}{l}
a_{1} \\
a_{2}
\end{array}\right)+\frac{4 \omega \zeta^{*}}{|\zeta|^{2}+\left|z_{1}\right|^{2}+\left|z_{2}\right|^{2}}\left(\begin{array}{c}
z_{1} \\
z_{2}
\end{array}\right)
$$



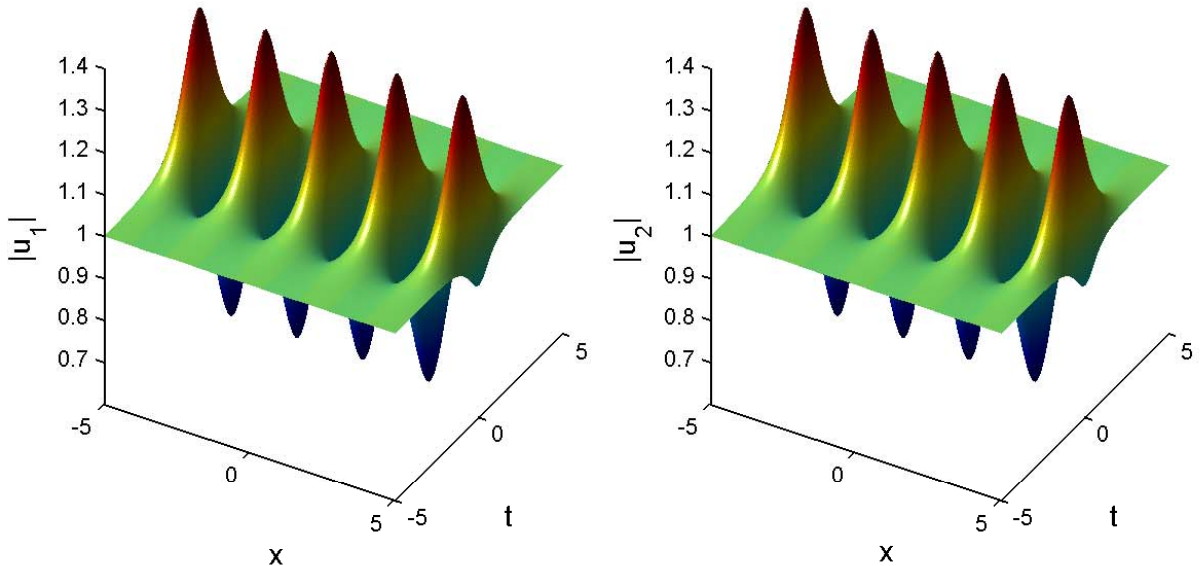

Figure 4: (Color online) Typical spatially periodic breather of the VNLSE (10 for parameters $a_{1}=1, a_{2}=1, \lambda=\frac{i}{4}, \mu_{1}=1, \mu_{2}=1, \mu_{3}=1$.
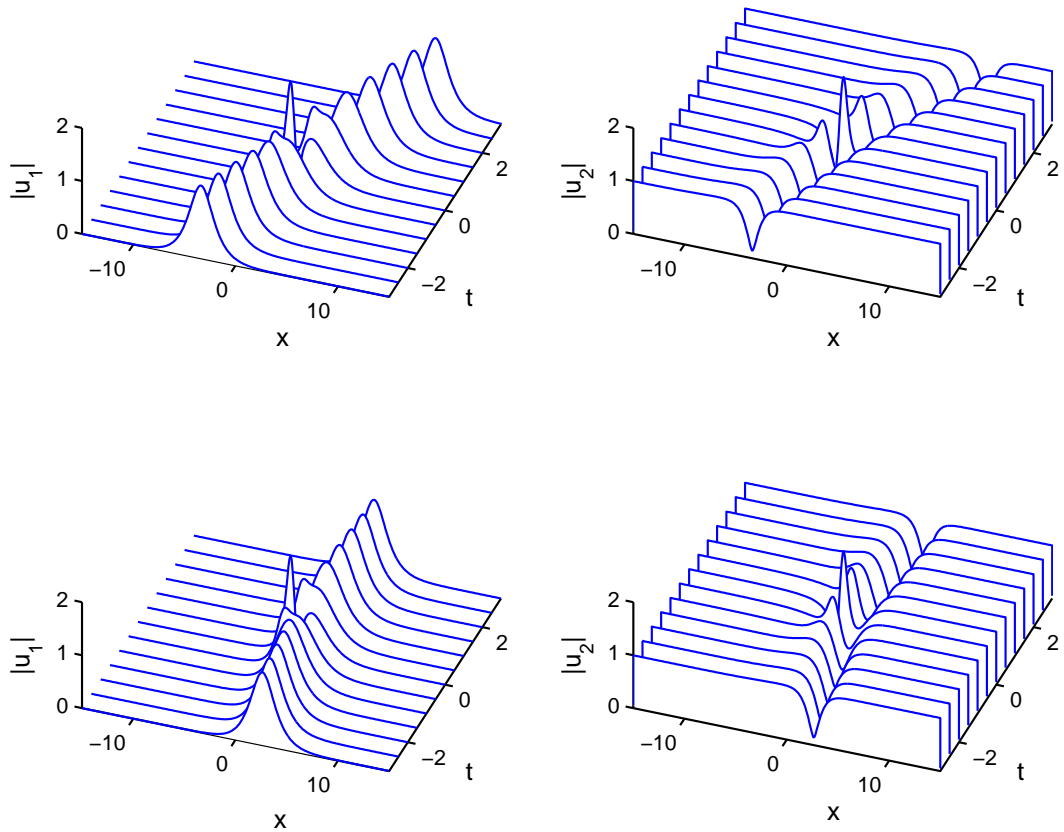

Figure 5: (Color online) Bright-dark rogue waves are emerging in the process of soliton propagation. The parameters are $a_{1}=0, a_{2}=1, \mu_{1}=1, \mu_{2}=1, \mu_{3}=\frac{1}{2}$. For wavenumber $k$, the top plane corresponds to $k=2$ while the bottom corresponds to $k=0$. 
where

$$
\begin{aligned}
& \left(\begin{array}{lll}
\zeta & z_{1} & z_{2}
\end{array}\right)^{T}=A F G Z_{0}, \quad Z_{0}=\left(\begin{array}{lll}
\mu_{1} & \mu_{2} & \mu_{3}
\end{array}\right)^{T}, \\
& F=\left(\begin{array}{ccc}
1-\omega x & -a_{1} x & -a_{2} x \\
a_{1} x & \omega^{-2}\left(a_{1}^{2} \varpi+a_{2}^{2} e^{\omega x}\right) & a_{1} a_{2} \omega^{-2}\left(\varpi-e^{\omega x}\right) \\
a_{2} x & a_{1} a_{2} \omega^{-2}\left(\varpi-e^{\omega x}\right) & \omega^{-2}\left(a_{2}^{2} \varpi+a_{1}^{2} e^{\omega x}\right)
\end{array}\right), \\
& G=\left(\begin{array}{ccc}
1+1 \mu_{0} \nu_{0} t / 2 & -a_{1} \nu_{0} t & -a_{2} \nu_{0} t \\
a_{1} \nu_{0} t & a_{1}^{2} \zeta_{1}+a_{2}^{2} \zeta_{2} & a_{1} a_{2}\left(\zeta_{1}-\zeta_{2}\right) \\
a_{2} \nu_{0} t & a_{1} a_{2}\left(\zeta_{1}-\zeta_{2}\right) & a_{2}^{2} \zeta_{1}+a_{1}^{2} \zeta_{2}
\end{array}\right)
\end{aligned}
$$

with $\varpi=1+\omega x, \zeta_{1}=\omega^{-2}\left(1-i \mu_{0} \nu_{0} t / 2\right), \zeta_{2}=\omega^{-2} e^{-i\left(\omega^{2} t+\mu_{0} \nu_{0} t / 2\right)}$ and $\mu_{0}=2 i \omega, \nu_{0}=$ $2 i \omega-2 k$. This is plotted in Figure 5 and illustrates how rogue waves can occur in the propagation of a bright-dark soliton. Note that the bright-dark soliton with a non-zero wavenumber has a slight change in velocities and phases when compared to the case with a zero wavenumber. Similar dynamic behaviour occurs for the other values of $a_{1}, a_{2}$ and $Z$, but the corresponding figures are omitted here.

\section{Derivation of $N$ th-order rogue waves solutions}

In this section, we derive $N$ th-order rogue waves of the VNLSE (1) using our expansion theorem of section 2. For simplicity, we consider only the case of zero wavenumber $k=0$. Thus we let $\lambda=i \omega(1+\delta)(0<\delta<1)$ in $(39)$. Using Taylor series expansions expansions for the trigonometric and exponential functions, the matrix $F$ in (43) can be expanded around $\delta=0$ as

$$
\left.F\right|_{\lambda=i \omega(1+\delta)}=\sum_{n=0}^{\infty} F_{n} \delta^{n}
$$

where

$$
F_{n}=\left(\begin{array}{ccc}
\vartheta_{n} & -a_{1} \omega^{-1} \beta_{n} & -a_{2} \omega^{-1} \beta_{n} \\
a_{1} \omega^{-1} \beta_{n} & \omega^{-2}\left(a_{1}^{2} \Pi_{n}+a_{2}^{2} e^{\omega x} A_{n}\right) & a_{1} a_{2} \omega^{-2}\left(\Pi_{n}-e^{\omega x} A_{n}\right) \\
a_{2} \omega^{-1} \beta_{n} & a_{1} a_{2} \omega^{-2}\left(\Pi_{n}-e^{\omega x} A_{n}\right) & \omega^{-2}\left(a_{2}^{2} \Pi_{n}+a_{1}^{2} e^{\omega x} A_{n}\right)
\end{array}\right),
$$

with

$$
\begin{aligned}
& \vartheta_{n}=\alpha_{n}-\beta_{n}-\beta_{n-1}, \quad \Pi_{n}=\alpha_{n}+\beta_{n}+\beta_{n-1}, \quad A_{m}=\frac{\omega^{m} x^{m}}{m !}, \\
& \alpha_{n}=\sum_{l=0}^{\lfloor n / 2\rfloor} C_{n-l}^{l} 2^{n-2 l} A_{2(n-l)}, \quad \beta_{n}=\sum_{l=0}^{\lfloor n / 2\rfloor} C_{n-l}^{l} 2^{n-2 l} A_{2(n-l)+1}, C_{n}^{m}=\frac{n !}{m !(n-m) !} .
\end{aligned}
$$


Here $n$ and $m$ are nonnegative integers with $n \geq m$. Similarly, the matrix $G$ in (44) has the expansion

$$
\left.G\right|_{\lambda=i \omega(1+\delta)}=e^{-i \omega^{2} t} \sum_{n=0}^{\infty} G_{n} \delta^{n}
$$

where

$$
G_{n}=\left(\begin{array}{ccc}
\sigma_{n} & -a_{1} \omega^{-1} \gamma_{n} & -a_{2} \omega^{-1} \gamma_{n} \\
a_{1} \omega^{-1} \gamma_{n} & \omega^{-2}\left(a_{1}^{2} \chi_{n}+a_{2}^{2} e^{i \omega^{2} t} \rho_{n}\right) & a_{1} a_{2} \omega^{-2}\left(\chi_{n}-e^{i \omega^{2} t} \rho_{n}\right) \\
a_{2} \omega^{-1} \gamma_{n} & a_{1} a_{2} \omega^{-2}\left(\chi_{n}-e^{i \omega^{2} t} \rho_{n}\right) & \omega^{-2}\left(a_{2}^{2} \chi_{n}+a_{1}^{2} e^{i \omega^{2} t} \rho_{n}\right)
\end{array}\right)
$$

with

$$
\begin{aligned}
\sigma_{n} & =\gamma_{n}-\theta_{n}-\theta_{n-1}, \quad \chi_{n}=\gamma_{n}+\theta_{n}+\theta_{n-1}, \\
\gamma_{n} & =\sum_{l=0}^{\lfloor 3 n / 4\rfloor} \sum_{m=0}^{l}(-1)^{n-l} C_{n-l}^{m} C_{2(n-l)}^{l-m} 2^{n-l-m} B_{2(n-l)}, \\
\theta_{n} & =1 \sum_{l=0}^{\lfloor(3 n+1) / 4\rfloor} \sum_{m=0}^{l}(-1)^{n-l} C_{n-l}^{m} C_{2(n-l)+1}^{l-m} 2^{n-l-m} B_{2(n-l)+1}, \\
\rho_{n} & =\sum_{l=0}^{\lfloor n / 2\rfloor} C_{n-l}^{l} i^{n-l} 2^{n-2 l} B_{n}, \quad B_{m}=\frac{2^{m} \omega^{2 m} t^{m}}{m !} .
\end{aligned}
$$

On the above formulas, $l$ should be a nonnegative integer. Next, let us assume $Z_{0}$ be an arbitrary polynomial function of $\delta$ given by

$$
Z_{0}(\delta)=\sum_{k=0}^{n} W_{k} \delta^{k}
$$

where $W_{k}=\left(\begin{array}{lll}\mu_{1 k} & \mu_{2 k} & \mu_{3 k}\end{array}\right)^{T}$ are arbitrary constant vectors. Thus,

$$
\left.\Psi\right|_{\lambda=i \omega(1+\delta)}=\sum_{n=0}^{\infty} \Psi_{n} \delta^{n}, \quad \Psi_{n}=e^{-i \omega^{2} t} A H_{n}, \quad H_{n}=\sum_{k=0}^{n} \sum_{j=0}^{n} F_{k} G_{j} W_{n-k-j},
$$

Then taking $\lambda_{1}=i \omega$ in the Theorem yields the $N$-rogue waves solutions of the VNLSE (1). Since the matrixes $F_{i}$ are independent of the variable $t$ while the matrixes $G_{i}$ are independent of the variable $x$, the $N$ th rogue wave solutions for VNLSE are expressed explicitly in a separation of variable form.

Moreover, if the components of the vector $W_{k}$ satisfy the conditions

$$
a_{1} \mu_{3 k}-a_{2} \mu_{2 k}=0, k=1 \cdots N,
$$

then solution (17) can be reduced to an $N$ th-order pure rational solution. In particular, when $N=1,2,3$, the solutions reduce to the cases considered by Zhai et al. 32 . 


\section{Dynamics of rogue wave solutions}

In this section, we discuss the dynamics of these high-order rogue-wave solutions. First setting $N=1$, the first-order rogue wave is produced,

$$
\begin{gathered}
\left(\begin{array}{l}
u_{11} \\
u_{21}
\end{array}\right)=e^{2 i \omega^{2} t}\left(\begin{array}{c}
a_{1} \\
a_{2}
\end{array}\right)+\frac{4 \omega r[0]^{*}}{|r[0]|^{2}+\left|s_{1}[0]\right|^{2}+\left|s_{2}[0]\right|^{2}}\left(\begin{array}{c}
s_{1}[0] \\
s_{2}[0]
\end{array}\right), \\
\text { where } \quad\left(r[0], s_{1}[0], s_{2}[0]\right)^{T} \equiv \psi[0]=\Psi_{0}=e^{-i \omega^{2} t} A F_{0} G_{0} W_{0}, \\
F_{0}=\left(\begin{array}{ccc}
1-\omega x & -a_{1} x & -a_{2} x \\
a_{1} x & \omega^{-2}\left(a_{1}^{2} \varpi+a_{2}^{2} e^{\omega x}\right) & a_{1} a_{2} \omega^{-2}\left(\varpi-e^{\omega x}\right) \\
a_{2} x & a_{1} a_{2} \omega^{-2}\left(\varpi-e^{\omega x}\right) & \omega^{-2}\left(a_{2}^{2} \varpi+a_{1}^{2} e^{\omega x}\right)
\end{array}\right), \\
G_{0}=\left(\begin{array}{ccc}
-2 i \omega^{2} t+1 & -2 i a_{1} \omega t & -2 i a_{2} \omega t \\
2 i a_{1} \omega t & a_{2}^{2} \omega^{-2} e^{i \omega^{2} t}+a_{1}^{2} \Gamma & a_{1} a_{2}\left(\Gamma-\omega^{-2} e^{i \omega^{2} t}\right) \\
2 a_{2} \omega i t & a_{1} a_{2}\left(\Gamma-\omega^{-2} e^{i \omega^{2} t}\right) & a_{1}^{2} \omega^{-2} e^{i \omega^{2} t}+a_{2}^{2} \Gamma
\end{array}\right),
\end{gathered}
$$

with the following notation: $\varpi=1+\omega x, \Gamma=\omega^{-2}+21 t$. This agrees with 46 in the case of $k=0$ and has been discussed in detail in the work [24].

Taking $N=2$, the second-order rogue wave is produced,

$$
\begin{aligned}
& \left(\begin{array}{c}
u_{12} \\
u_{22}
\end{array}\right)=\left(\begin{array}{c}
u_{11} \\
u_{21}
\end{array}\right)+\frac{4 \omega r[1]^{*}}{|r[1]|^{2}+\left|s_{1}[1]\right|^{2}+\left|s_{2}[1]\right|^{2}}\left(\begin{array}{c}
s_{1}[1] \\
s_{2}[1]
\end{array}\right), \\
& \text { where } \quad\left(r[1] s_{1}[1] \quad s_{2}[1]\right)^{T} \equiv \psi[1]=21 \omega\left[(I-P[1]) \Psi_{1}+\frac{1}{2} \psi_{0}\right], \\
& P[1]=\frac{\psi[0] \psi[0]^{\dagger}}{\psi[0]^{\dagger} \psi[0]}, \quad \Psi_{1}=e^{-i \omega^{2} t} A\left[\left(F_{0} G_{1}+F_{1} G_{0}\right) W_{0}+F_{0} G_{0} W_{1}\right],
\end{aligned}
$$

The matrixes $F_{1}$ and $G_{1}$ are given by

$$
\begin{gathered}
F_{1}=\left(\begin{array}{ccc}
-\omega x\left(1-\omega x+\frac{\omega^{2} x^{2}}{3}\right) & -\frac{1}{3} a_{1} \omega^{2} x^{3} & -\frac{1}{3} a_{2} \omega^{2} x^{3} \\
\frac{1}{3} a_{1} \omega^{2} x^{3} & a_{1}^{2} H+a_{2}^{2} \omega^{-1} x e^{\omega x} & a_{1} a_{2}\left(H-\omega^{-1} x e^{\omega x}\right) \\
\frac{1}{3} a_{2} \omega^{2} x^{3} & a_{1} a_{2}\left(H-\omega^{-1} x e^{\omega x}\right) & a_{2}^{2} H+a_{1}^{2} \omega^{-1} x e^{\omega x}
\end{array}\right), \\
G_{1}=\left(\begin{array}{ccc}
-4 \omega^{2} t\left(i+\omega^{2} t-\frac{2}{3} i \omega^{4} t^{2}\right) & \frac{2}{3} i a_{1} \omega t\left(-3+4 \omega^{4} t^{2}\right) & \frac{2}{3} i a_{2} \omega t\left(-3+4 \omega^{4} t^{2}\right) \\
\frac{2}{3} i a_{1} \omega t\left(3-4 \omega^{4} t^{2}\right) & a_{1}^{2} J+4 i a_{2}^{2} t e^{i \omega^{2} t} & a_{1} a_{2}\left(J-4 i t e^{i \omega^{2} t}\right) \\
\frac{2}{3} i a_{2} \omega t\left(3-4 \omega^{4} t^{2}\right) & a_{1} a_{2}\left(J-4 i t e^{i \omega^{2} t}\right) & a_{2}^{2} J+4 i a_{1}^{2} t e^{i \omega^{2} t}
\end{array}\right),
\end{gathered}
$$


with the following notation: $H=\omega^{-1} x+x^{2}+\frac{\omega x^{3}}{3}$ and $J=4 t\left(i-\omega^{2} t-\frac{2}{3} i \omega^{4} t^{2}\right)$.

If the conditions $a_{1} \mu_{30}-a_{2} \mu_{20}=0$ and $a_{1} \mu_{31}-a_{2} \mu_{21}=0$ are imposed, the expression (52) yields a trival generalisation of the rational Peregrine solution. This is the situation considered in the work [32. In this case, the component $u_{1}(x, t)$ is merely proportional to $u_{2}(x, t)$. Here, we are concerned with other parameter values.

Figures 6 to 10 illustrate four different kinds of spatial-temporal distribution patterns for these second order solutions (52) for different parameter values. In figure 6 under the condition that the vector $W_{1}=0$, we see that two solitons and a second-order rogue wave coexist. The second-order rogue wave in $\left|u_{1}(x, t)\right|$ has a peak whose amplitude is about 3 and reaches 4 times that of the background plane wave amplitude in $\left|u_{2}(x, t)\right|$. In figure 7, we set $W_{1}=-W_{0}$, and then the component $u_{1}(x, t)$ in the second-order rogue wave is difficult to see, while the other component $u_{2}(x, t)$ approaches 5 times that of the background plane wave amplitude. This shows that the amplitudes of the peaks of the second-order rogue wave depend on the values of these free parameters. This can be observed more clearly in Figure 8. Similarly to the first-order rogue wave, if the parameter $a_{1} \neq 0$ ias shown in Figure 9, both the two bright solitons, and the two dark solitons display breathing behaviour. Figure 10 shows that five intensity humps appear at different times and/or space. In Figures 11 to 13 we show some typical examples of the third-order rogue waves coexisting with three solitons. These third-order rogue waves have even higher amplitudes, as has been found for the single-component NLSE equation in Ref. [7],[14]-[16],[34]. By adjusting the values of the free parameters, more such interesting spatial-temporal patterns can be found.

These results shown above can be extended naturally to fourth-, and even higher-order rogue waves. We find that the higher-order rogue waves can be obtained most easily if we only consider the parameter $W_{0}$. Moreover, if all the parameters $a_{1}, a_{2}, W_{i}$ are nonzero, the soliton-like waves perform breathing behaviour. In addition, multiple intensity humps appear at different times and/or space provided the vector $W_{i}$ is not proportional to the vector $W_{j}$ for $i \neq j$. Finally, we note that the velocities of the multisoliton solutions generally depend on time, just like the one-soliton case studied by Baronio et al. [24] The dynamic behaviours found here could be interpreted as a mechanism to excite rogue wave with higher amplitude out of multiple slowly moving breathing solitons.

\section{Conclusion}

In this paper we have shown how to construct new higher-order rogue waves in the vector nonlinear Schrödinger equations (Manakov system) (1) by using a Darboux-dressing transformation combined with an asymptotic expansion. These $N$ th-order rogue wave solutions contain $3 N$ parameters, and can be expressed explicitly in a separation of variable form. The found solutions exhibit a range of interesting and complicated dynamics, obtained by varying the available parameters. These include bright-dark higher-order rogue waves, bright-dark multi-rogue waves patterns, and rogue waves interacting with 

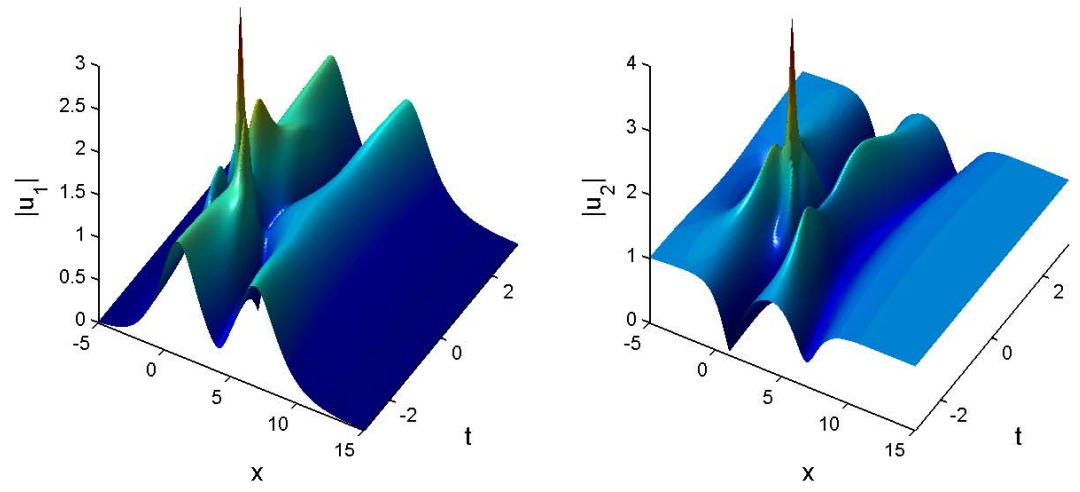

Figure 6: (Color online) Second order rogue waves emerging in the propagation of two bright-dark solitons. The parameters are $a_{1}=0, a_{2}=1, \mu_{10}=1, \mu_{20}=1, \mu_{30}=1, \mu_{11}=$ $0, \mu_{21}=0$ and $\mu_{31}=0$.
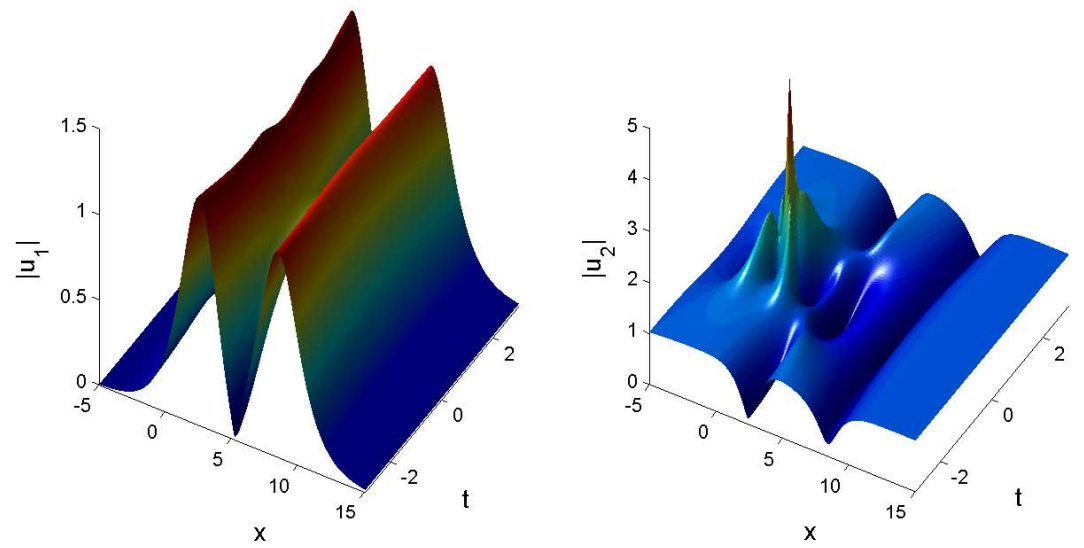

Figure 7: (Color online) Second order rogue waves emerging in the propagation of two bright-dark solitons. The parameters are $a_{1}=0, a_{2}=1, \mu_{10}=1, \mu_{20}=1, \mu_{30}=100, \mu_{11}=$ $-1, \mu_{21}=-1$ and $\mu_{31}=-100$. 

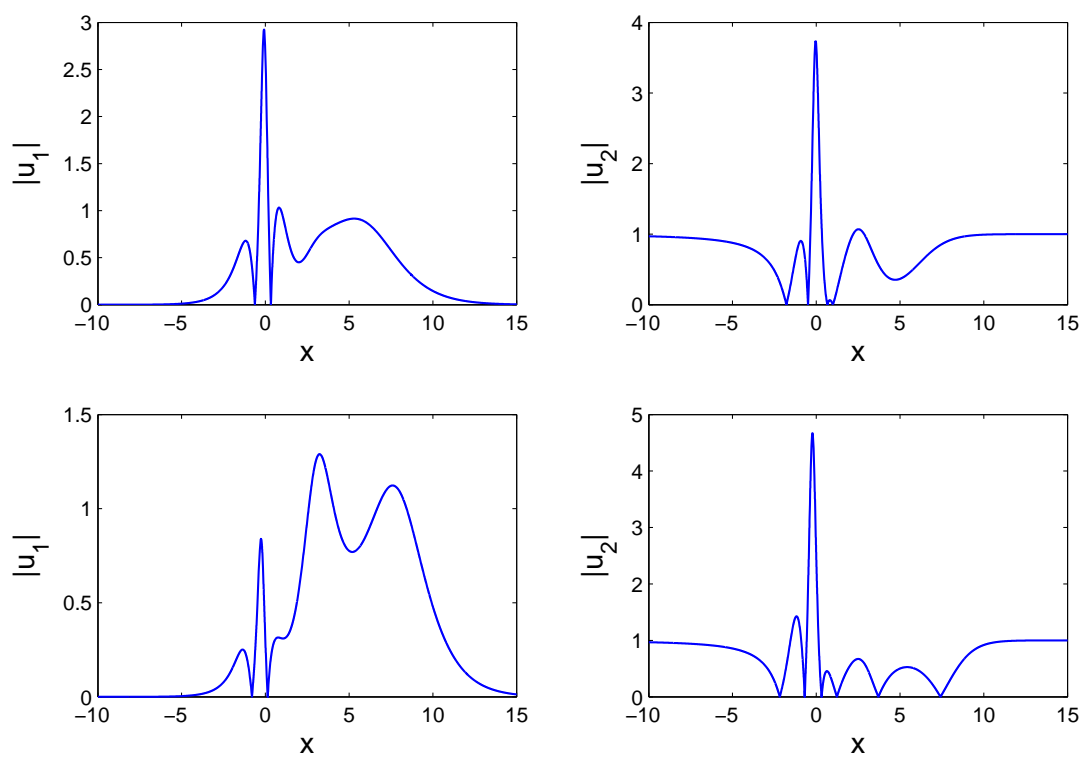

Figure 8: (Color online) Plots of the amplitudes of the functions $u_{1}(x, 0), u_{2}(x, 0)$ in Figures 5 and 6 .
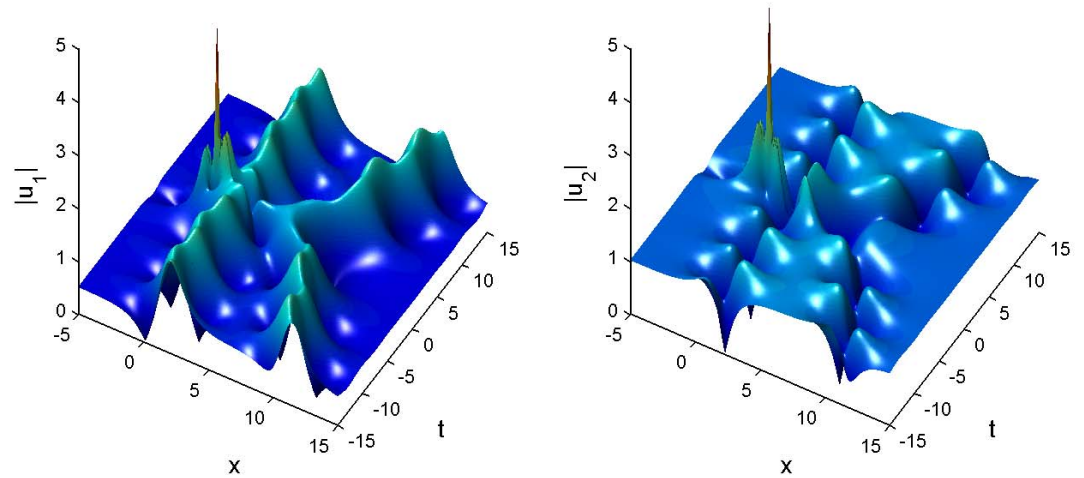

Figure 9: (Color online) Second order rogue waves emerging in the propagation of two breathing bright-dark solitons. The parameters are $a_{1}=\frac{1}{2}, a_{2}=1, \mu_{10}=1, \mu_{20}=1, \mu_{30}=$ $1, \mu_{11}=0, \mu_{21}=0$ and $\mu_{31}=0$. 

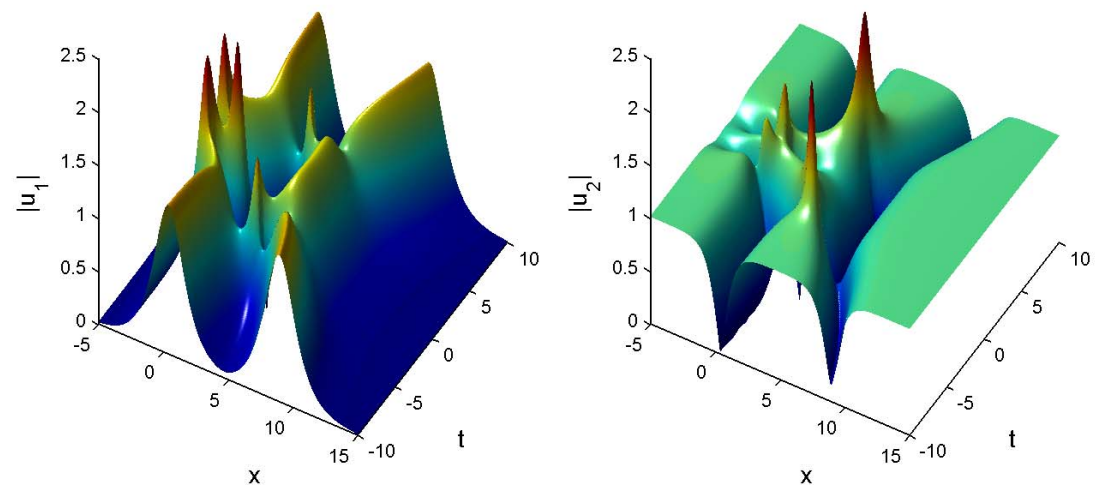

Figure 10: (Color online) Muti-rogue wave patterns emerging in the propagation of two bright-dark solitons. The parameters are $a_{1}=0, a_{2}=1, \mu_{10}=1, \mu_{20}=1, \mu_{30}=0, \mu_{11}=$ $1, \mu_{21}=1$ and $\mu_{31}=15$.
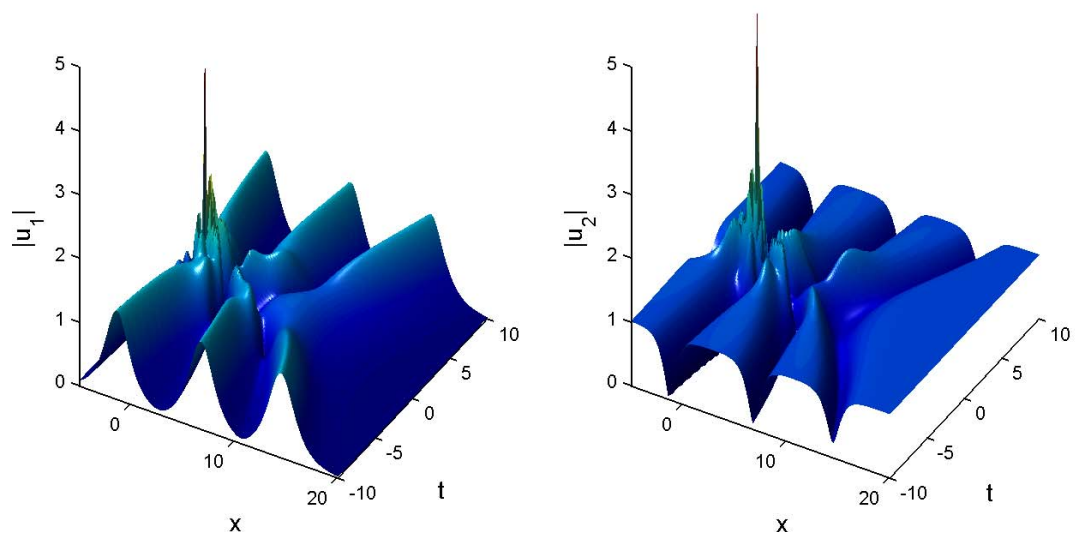

Figure 11: (Color online) Third order rogue wave patterns emerging in the propagation of three bright-dark solitons. The parameters are $a_{1}=0, a_{2}=1, \mu_{10}=1, \mu_{20}=1, \mu_{30}=$ $1, \mu_{11}=0, \mu_{21}=0, \mu_{31}=0, \mu_{12}=0, \mu_{22}=0$ and $\mu_{32}=0$. 

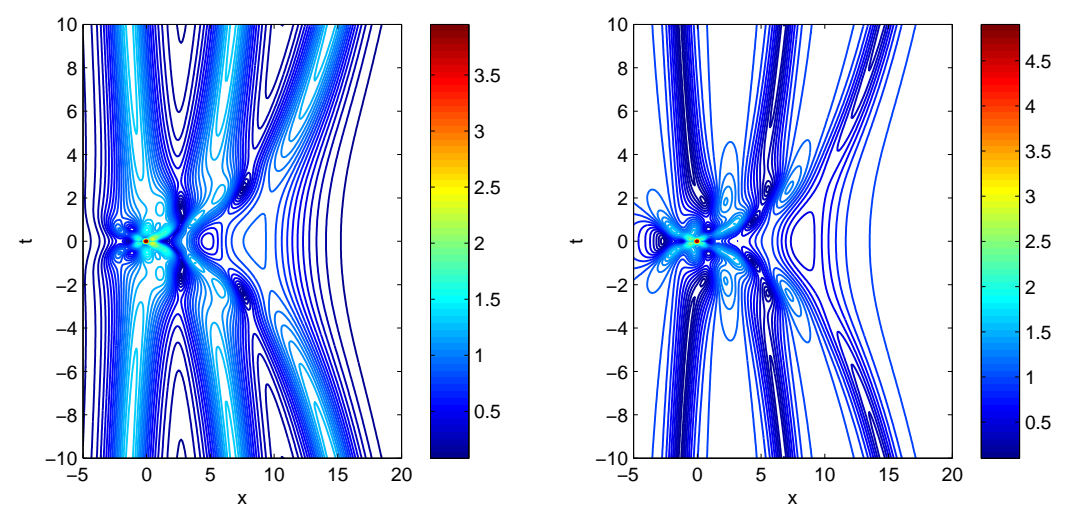

Figure 12: (Color online) Third order rogue waves emerging in the propagation of three bright-dark solitons. The parameters are $a_{1}=0, a_{2}=1, \mu_{10}=1, \mu_{20}=1, \mu_{30}=1, \mu_{11}=$ $0, \mu_{21}=0, \mu_{31}=0, \mu_{12}=0, \mu_{22}=0$ and $\mu_{32}=0$.
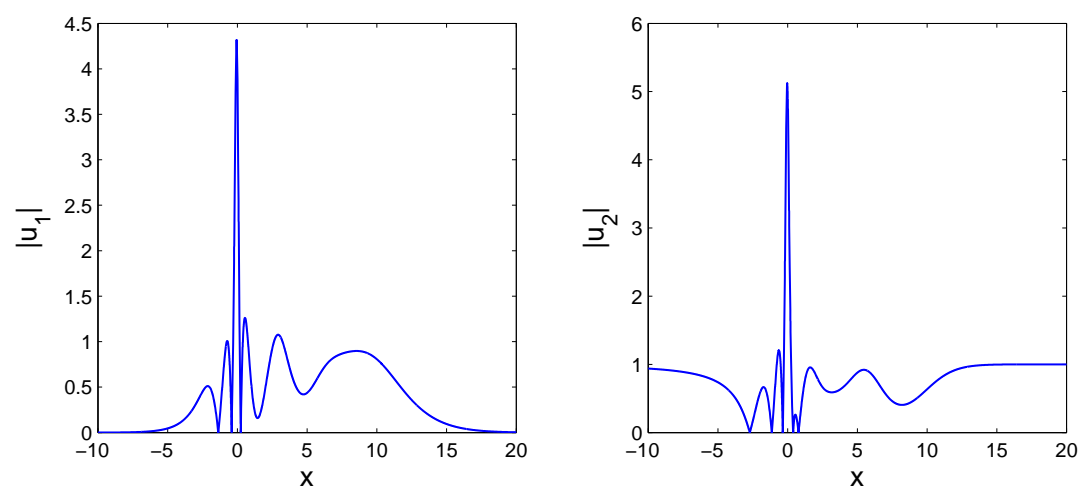

Figure 13: (Color online) Plots of the amplitudes of the functions $u_{1}(x, 0), u_{2}(x, 0)$ in Figure 11 . 
multisolitons. These new spatial-temporal patterns reveal the potential rich dynamics in rogue wave solutions, and although found here in a VNLSE system, rather than in the usual single-component NLSE, promote our understanding of rogue-wave phenomena.

Although our solutions exhibited here have set the wavenumber $k=0$, the same procedure can be used when $k \neq 0$. One begins again with the solution $(39)$ and the following analysis is only slightly more complex. Further, since in a vector system modulational instability may occur in the normal dispersion regime resulting from cross-phase modulation, the corresponding rogue wave solutions are of interest, and the technique presented in this paper is also available for Matrix nonlinear Schrödinger equation[21], these will be reported elsewhere. Also, the novel connection between mulitsolitons and rogue waves revealed here has potentially far-reaching significance, and a topic we will explore in the future.

\section{Acknowledgments}

The work was supported in part by the National Natural Science Foundation of China (No. 10801037), the New Teacher Grant of Ministry of Education of China (No. 200802461007), the Young Teachers Foundation (No. 1411018) of Fudan university and Yunnan province project Education Fund: No. 2013C012. Also, the authors are very grateful to Professor Peter D. Miller, Professor John E. Fornaess and Professor Zhengde Dai for their enthusiastic support and useful suggestions.

\section{References}

[1] C. Kharif, E. Pelinovsky and A. Slunyaev, Rogue waves in the ocean, "Advances in Geophysical and Environmental Mechanics and Mathematics", vol 14, Springer: Berlin, 2009, pp. 216.

[2] A. R. Osborne, "Nonlinear ocean waves and the inverse scattering transform", Elsevier: Amsterdam, The Netherlands, 2010, pp. 914.

[3] A. Slunyaev, I. Didenkulova and E. Pelinovsky, Rogue waters, Contemp. Phys., 52 (2011), pp. 571-590.

[4] B. Kibler, J. Fatome, C. Finot, G. Millot, F. Dias, G. Genty, G., N. Akhmediev and J. M. Dudley, The Peregrine soliton in nonlinear fibre optics, Nat. Phys., 6 (2010), pp. $790-795$.

[5] B. Kibler, J. Fatome, C. Finot, G. Millot, G. Genty, B. Wetzel, N. Akhmediev, F. Dias and J. M. Dudley, Observation of Kuznetsov-Ma soliton dynamics in optical fibre, Sci. Rep., 2 (2012), 463. 
[6] D. R. Solli, C. Ropers, P. Koonath and B. Jalali, Optical rogue waves, Nature, 450 (2007), pp. 1054-1057.

[7] N. Akhmediev, J.M. Soto-Crespo and A. Ankiewicz, Extreme waves that appear from nowhere: On the nature of rogue waves, Phys. Lett. A, 373 (2009), pp. 2137-2145.

[8] V. E. Zakharov and A. B. Shabat, Exact theory of two-dimensional self-focusing and one-dimensional self-modulation of waves in nonlinear media, Sov. Phys. JETP, 34 (1972), pp. 62-69.

[9] D. H. Peregrine, Water waves, nonlinear Schrödinger equations and their solutions, J. Aust. Math. Soc. B, 25 (1983), pp. 16-43.

[10] E. Kuznetsov, Solitons in a parametrically unstable plasma, Sov. Phys. Dokl., 22 (1977), pp. 507-508.

[11] Y. C. Ma, The perturbed plane-wave solutions of the cubic Schröinger equation, Stud. Appl. Math., 60 (1979), pp. 43-58.

[12] R. Grimshaw and A. Tovbis, Rogue waves: Analytical predictions, Proc. Royal Soc., 469 (2013), 20130094.

[13] A. Chabchoub, N. P. Hoffmann, and N. Akhmediev, Rogue Wave Observation in a Water Wave Tank, Phys. Rev. Lett., 106 (2011), 204502.

[14] N. Akhmediev, A. Ankiewicz and M. Taki, Waves that appear from nowhere and disappear without a trace, Phys. Lett. A, 373 (2009), pp. 675-678.

[15] Y. Ohta and J. Yang, General high-order rogue waves and their dynamics in the nonlinear Schrödinger equation, Proc. Roy. Soc. A, 468 (2012), pp. 1716-1740.

[16] B. L. Guo, L. M. Ling, and Q. P. Liu, Nonlinear Schrödinger equation: Generalized Darboux transformation and rogue wave solutions, Phys. Rev. E, 85 (2012), 026607.

[17] B. L. Guo, L. M. Ling and Q. P. Liu, High-Order Solutions and Generalized Darboux Transformations of Derivative Nonlinear Schrödinger Equations, Stud. Appl. Math., 130 (2013), pp. 317-344.

[18] P. Dubard, P. Gaillard, C. Klein, and V. B. Matveev, On multi-rogue wave solutions of the NLS equation and positon solutions of the KdV equation, Eur. Phys. J. Spec. Top., 185 (2010), pp. 247-258.

[19] A. Ankiewicz, N. Akhmediev and J. M. Soto-Crespo, Discrete rogue waves of the Ablowitz-Ladik and Hirota equations, Phys. Rev. E, 82 (2010), 026602.

[20] J. E. He, S. W. Xu and K. Porsezian, New Types of Rogue Wave in an Erbium-Doped Fibre System, J. Phys. Soc. Jpn., 81 (2012), 033002. 
[21] Z. Y. Qin and G. Mu, Matter rogue waves in an $\mathrm{F}=1$ spinor Bose-Einstein condensate, Phys. Rev. E, 86 (2012), 036601.

[22] G. Mu and Z. Y. Qin, Rogue Waves for the Coupled Schrödinger-Boussinesq Equation and the Coupled Higgs Equation, J. Phys. Soc. Jpn., 81 (2012), 084001.

[23] G. Mu and Z. Y. Qin, Two spatial dimensional N-rogue waves and their dynamics in Melnikov equation, Nonlinear Anal. Real World Appl., 18 (2014), pp. 1-13.

[24] F. Baronio, A. Degasperis, M. Conforti and S. Wabnitz, Solutions of the Vector Nonlinear Schrödinger Equations: Evidence for Deterministic Rogue Waves, Phys. Rev. Lett., 109 (2012), 044102.

[25] D. J. Kaup, B. A. Malomed, B. A. and R. S. Tasgal, Internal dynamics of a vector soliton in a nonlinear optical fiber, Phys. Rev. E, 48 (1993), 3049.

[26] J. M. Bilbault, P. Marquié and B. Michaux, Modulational instability of two counterpropagating waves in an experimental transmission line, Phys. Rev. E, 51 (1995), pp. 817-820.

[27] M. G. Forest, D. W. McLaughlin, D. J. Muraki, and O. C. Wright, 2000 Nonfocusing Instabilities in Coupled, Integrable Nonlinear Schrödinger pdes, J. Nonlinear Sci. 10 (2000), pp. 291-331.

[28] Y. V. Bludov, V. V. Konotop and N. Akhmediev, Vector rogue waves in binary mixtures of Bose-Einstein condensates, Eur. Phys. J. Special Topics, 185 (2010), pp. 169-180.

[29] N. Berloff, Solitary Wave Complexes in Two-Component Condensates, Phys. Rev. Lett., 94 (2005), 120401.

[30] K. Kasamatsu and M. Tsubota, Multiple Domain Formation Induced by Modulation Instability in Two-Component Bose-Einstein Condensates, Phys. Rev. Lett., 93 (2004), 100402.

[31] B. L. Guo and L. M. Ling, 2011 Rogue Wave, Breathers and Bright-Dark-Rogue Solutions for the Coupled Schrödinger Equations, Chin. Phys. Lett., 28 (2011), 110202.

[32] B. G Zhai, W. G. Zhang, X. L. Wang and H. Q. Zhang, Multi-rogue waves and rational solutions of the coupled nonlinear Schrödinger equations, Nonlinear Anal. Real World Appl., 14 (2013), pp. 14-27.

[33] A. Degasperis and S. Lombardo, Multicomponent integrable wave equations: I. Darboux-dressing transformation, J. Phys. A: Math. Theor., 40 (2007), pp. 961-977. 
[34] D. J. Kedziora, A. Ankiewicz and N. Akhmediev, Classifying the hierarchy of nonlinear-Schrödinger-equation rogue-wave solutions, Phys. Rev. E, 88 (2013), 013207. 